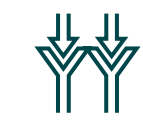

Endocrinology

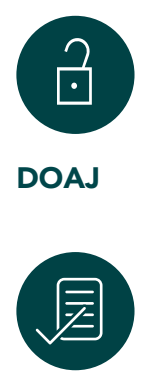

Acceptance rate $33 \%$

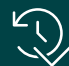

Time to final decision 7 weeks

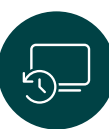

Time manuscript received to accepted 19 weeks

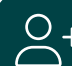

New Associate Editors

editorialoffice_dde@

karger.com

karger.com/dde

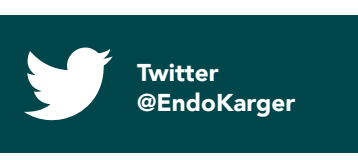

facebook.com/ EndoKarger

\section{Dubai Diabetes and Endocrinology Journal}

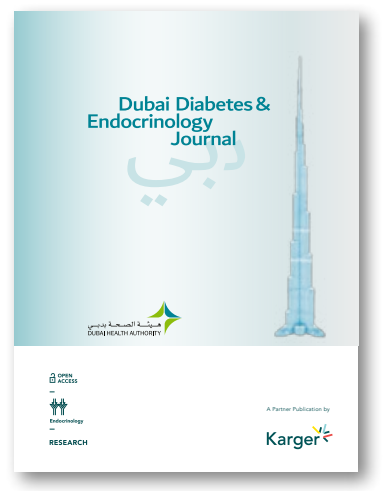

\author{
2 OPEN \\ 1 ACCESS
}

A Partner Publication by Karger

Editor-in-Chief

Fatheya Al Awadi (Dubai)

A global source of information to stay on top of scientific developments

The Dubai Diabetes and Endocrinology Journal welcomes original contributions on all aspects of diabetes, endocrinology, and metabolism. Taking into account that diabetes has become a pandemic, the journal provides a platform for worldwide communication and scientific exchange to researchers and clinicians dealing with diabetes. The focus of the journal lies on the publication of papers on morphological, biochemical, physiological, pathophysiological, and clinical aspects of research on diabetes and metabolism. In addition to research articles, further types of papers such as review articles, case reports, reviews, meta-analyses, letters, editorials, and commentaries make the journal a comprehensive and interesting read. The researcher and practitioner who seeks to stay up to date on the developments in the fields of diabetes and metabolism will find a wealth of information in the Dubai Diabetes and Endocrinology Journal. 